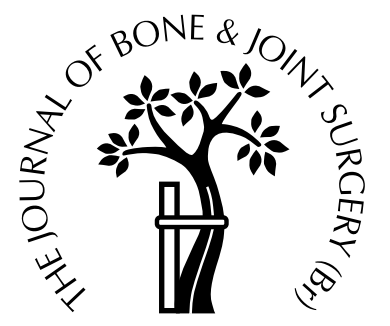

\title{
Patterns of loosening of the glenoid component
}

\author{
J. Nagels, E. R. Valstar, M. Stokdijk, P. M. Rozing \\ From Leiden University Medical Centre, The Netherlands
}

$T^{1}$ he incidence of loosening of a cemented glenoid component in total shoulder arthroplasty, detected by means of radiolucent lines or positional shift of the component on true anteroposterior radiographs, has been reported to be between $0 \%$ and $44 \%$.

Radiolucent lines are, however, difficult to detect and to interpret because of the mobility of the shoulder girdle and the obliquity of the glenoid which hinder standardisation of radiographs. We examined radiolucencies around cemented glenoid components in 48 patients, with a mean follow-up of 5.3 years, and found progressive changes to be present predominantly at the inferior pole of the component. This may hold a clue for the mechanism of loosening of this implant.

In five patients we performed an additional analysis of loosening of the glenoid component using digital roentgen stereophotogrammetric analysis (RSA). After three years, three of the five implants had loosened (migration 1.2 to $5.5 \mathrm{~mm}$ ). In only one, with gross loosening, were the radiological signs consistent with the RSA findings.

When traditional radiographs are used for assessment, the rate of early loosening is underestimated. We recommend that RSA be used for this.

J Bone Joint Surg [Br] 2002;84-B:83-7.

Received 1 December 2000; Accepted after revision 14 May 2001

J. Nagels, MD, PhD, Student

E. R. Valstar, PhD, Post-doctoral Researcher

M. Stokdijk, MSc, PhD, Student

P. M. Rozing, MD, PhD, Professor of Orthopaedics, Head of Department

Orthopaedic Laboratory, Department of Orthopaedics, Leiden University

Medical Centre, B0-57 PO Box 9600, 2300 RC Leiden, The Netherlands.

Correspondence should be sent to Dr J. Nagels.

(C)2002 British Editorial Society of Bone and Joint Surgery 0301-620X/02/111951 \$2.00
Total shoulder replacement is a satisfactory treatment for the painful and severely arthritic glenohumeral joint although there has been some dispute about its long-term outcome. One concern is mechanical loosening of the glenoid component. A positional shift or rotation of a component on plain radiographs is a firm indication of loosening. These changes are often seen in the long term, although the components may have been loose for quite some time. When assessing early loosening, the nature of any radiolucent lines present is commonly used. For glenoid components the overall prevalence of these radiolucent lines is reported to range from $22 \%$ to $95 \% .^{1-5}$ A review of the literature shows that the incidence of radiological loosening of glenoid components varies between $0 \%$ and $15 \%$ after follow-up for three years, ${ }^{2,3,6-8}$ rising to between $24 \%$ and $44 \%$ after nine years. ${ }^{4,5}$ Several authors have stressed the importance of a better insight into loosening ${ }^{2,6,10}$ since this can have serious adverse effects (e.g., dislocation of the component, osteolysis of the glenoid, glenohumeral instability) and may be a major factor in the long-term functional outcome. ${ }^{11}$

Detection of early loosening of the glenoid component requires an accurate and reproducible technique of measurement. $^{6,8,12}$ In 1974, Selvik ${ }^{13}$ developed roentgen stereophotogrammetric analysis (RSA) for measuring migration of prostheses. It is possible to measure the position of rigid bodies in three dimensions with a high degree of accuracy, ranging from 0.1 to $0.7 \mathrm{~mm}$. As a result small study groups and a short follow-up are sufficient to obtain an objective view on the success of fixation of implants. ${ }^{14,15}$ In order to obtain a better insight into the rate and the mechanism of loosening of a glenoid component and the role which radiolucencies play in the loosening process we have reviewed our radiological data and undertaken a pilot study using RSA.

\section{Patients and Methods}

Radiological assessment. Between 1984 and 1997, 56 patients received a total shoulder arthroplasty in our hospital with a cemented, polyethylene, keeled, glenoid component (Biomet Inc, Warsaw, Indiana). Disabling pain and corresponding radiological signs of degeneration had been the indications for replacement of the shoulder. At followup, seven patients had died and six others had been lost. Of 


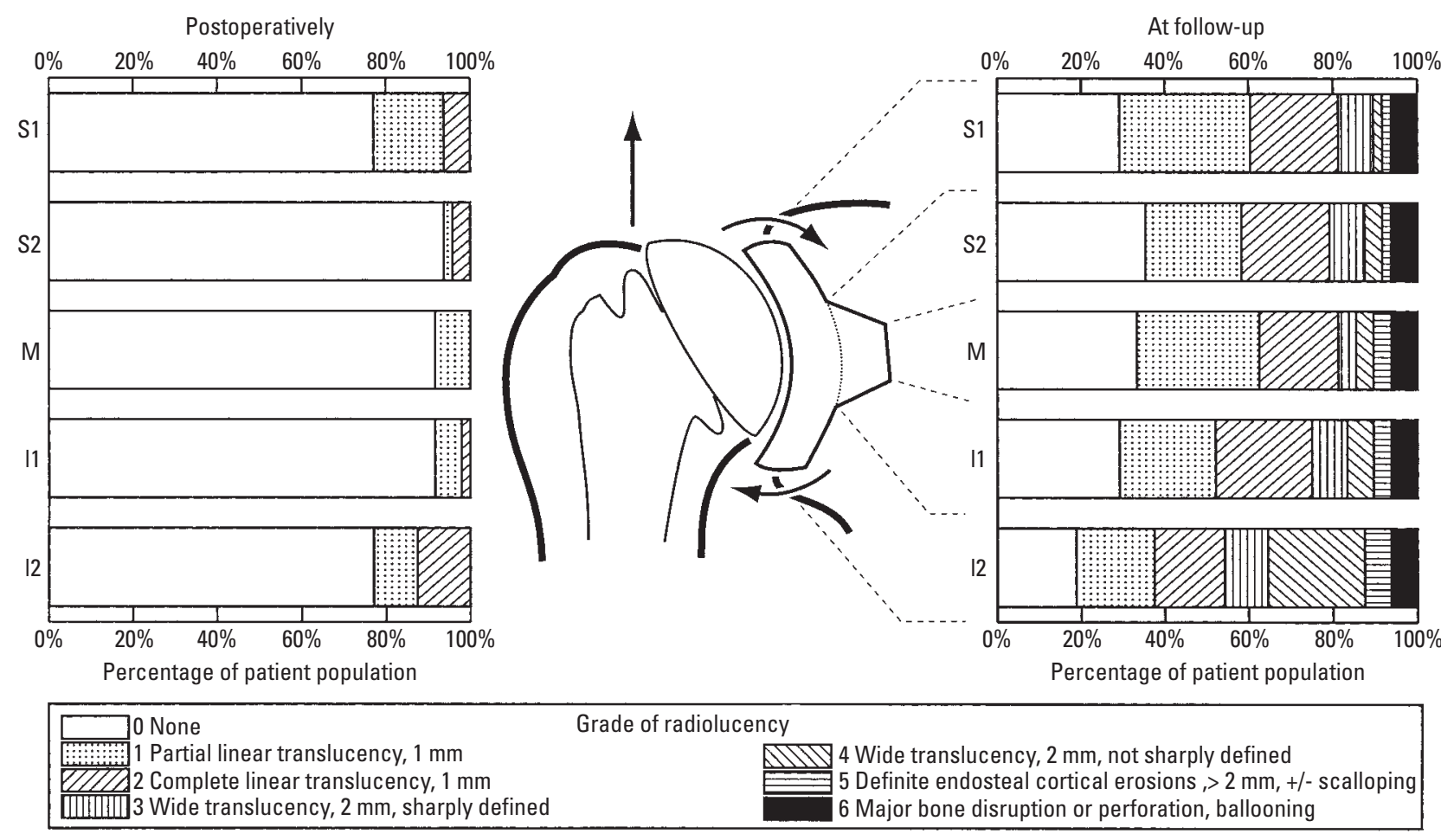

Fig. 1

Distribution of radiolucency about the glenoid component, observed immediately after operation and after a mean follow-up of 5.3 years in five different zones (S1, S2; superior parts; M, medial part; I1, I2; inferior parts). The arrows indicate the direction of the forces in the suggested mechanism of loosening which resulted in the observed pattern of radiolucency. The grading of radiolucency is according to a modification of Souter's score.

these 56 patients, these with a radiological record of over two years formed the basis for the study group which consisted of 48 patients (11 men and 37 women) with a mean age of 70.6 years ( 30 to 92 ). The underlying disease was rheumatoid arthritis in 36 patients and osteoarthritis in 12. The senior author (PMR) had operated on all patients.

After surgery the patients were reviewed in the outpatient clinic. The mean follow-up was for 5.3 years (2.1 to 10.6). At each visit a true anteroposterior (AP) radiograph was taken. While in an upright position, the patient was positioned with their back to the film, and rotated through $45^{\circ}$, so that the glenohumeral joint was projected as a line. The immediately postoperative radiograph was compared with the most recent film. The cement-bone interface was divided into five separate areas, from cranial to caudal, similar to the method used by Amstutz, Sew Hoy and Clarke. ${ }^{16}$ Each area was scored separately, using a modification of Souter's system ${ }^{17}$ (Fig. 1). Two independent observers reviewed the radiographs separately and discussed their analyses until both had agreed a final score for each area. Radiological loosening was defined as a progressive translucency around the glenoid component of $2 \mathrm{~mm}$ or more, spanning the whole cement-bone interface, or an apparent shift of the component.

RSA. Of the 48 patients, five ( 4 women and 1 man) with a mean age of 69 years (46 to 78 ) had RSA. Rheumatoid arthritis was present in four and one had primary osteoarthritis of the glenohumeral joint.

During surgery, at least three tantalum markers with a diameter of $1 \mathrm{~mm}$ were introduced into the collum scapulae. Given the limited amount of trabecular bone in the scapula, the markers were introduced into the neck of the scapula and the acromion as widely apart as possible. This ensured that, despite possible overprojection by the humeral head, enough markers would remain in view for RSA measurements. Another tantalum marker was placed in a drill hole in the keel of the polyethylene glenoid component. The latter was cemented after careful preparation and irrigation of the glenoid fossa. RSA radiographs were taken postoperatively, at six weeks, three and six months and at one, two and three years. The RSA apparatus consisted of two x-ray tubes, one uniplanar calibration box and a film cassette. The patient's shoulder was centred above the calibration box while in a supine position. Synchronised $\mathrm{x}$ ray tubes were positioned in such a way that the film-focus distance was $1.4 \mathrm{~m}$ and the centre of their beams crossed at the level of the shoulder, at an angle of $20^{\circ}$ to the vertical. Both views were projected on halves of a large x-ray film, located underneath the calibration box. The radiographs were digitised using the Vidar VXR-12 digitiser (Vidar, Lund, Sweden). Using the dedicated software package RSA-CMS (MEDIS, Leiden, The Netherlands), ${ }^{18,19}$ any 


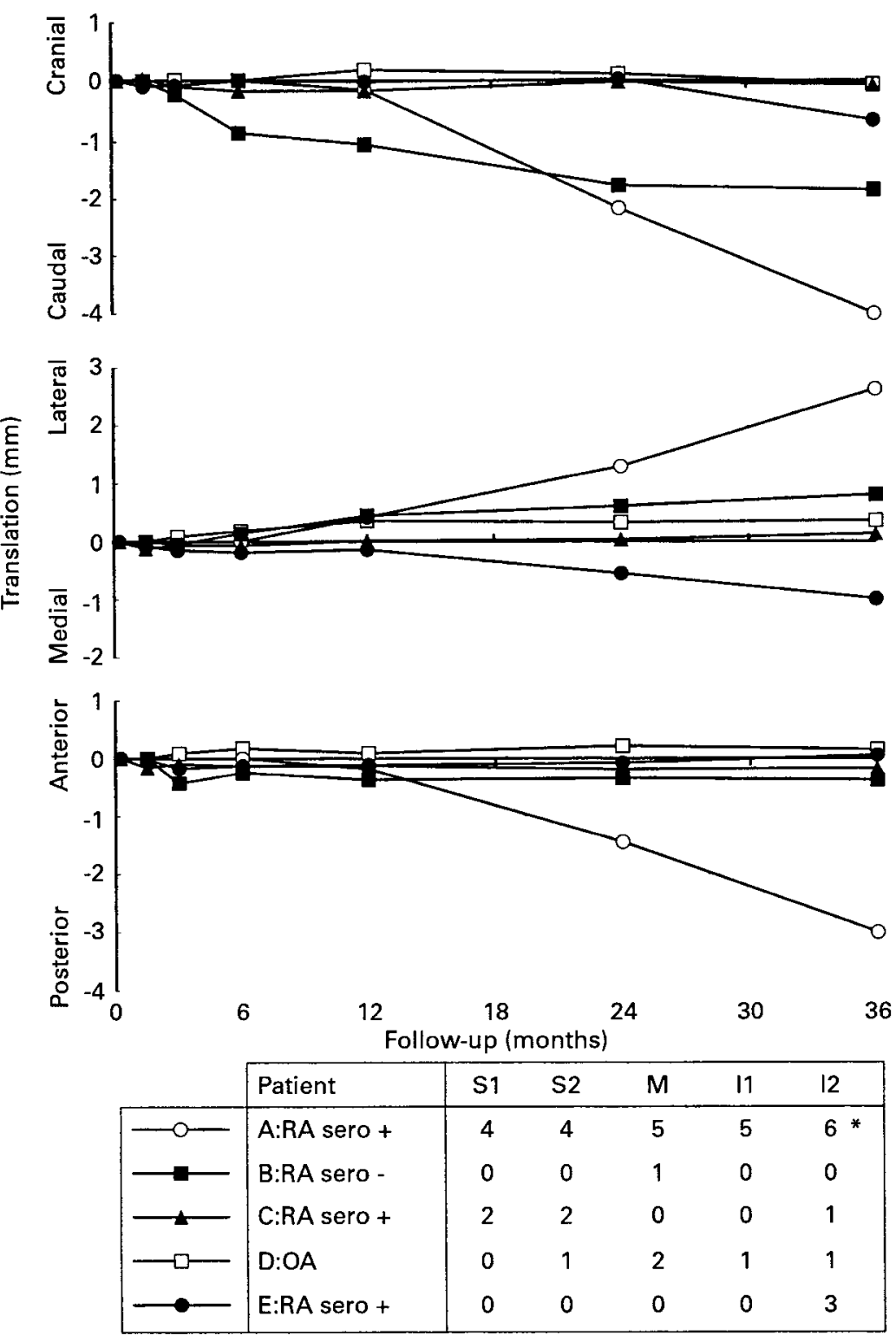

Fig. 2

The translations of the keel marker of the glenoid component with respect to the scapula are presented in cranial/caudal, medial/lateral and anterior/posterior directions during the three-year follow-up. The Table below the Figure shows the grades of radiolucency observed in the five different zones about the glenoid component after follow-up for 36 months in the RSA patients (for definition of grades and zones see Figure 1) (OA, osteoarthritis; RA, rheumatoid arthritis; * radiological loosening).

change in the position of the glenoid component, with respect to the scapular markers, was calculated in three dimensions. From sequential RSA radiographs, the relative movement of the glenoid component with respect to the scapula could be assessed.

For accuracy, two RSA examinations were undertaken for each of the five patients and the difference was calculated. The mean errors were $0.03 \pm 0.028 \mathrm{~mm}, 0.06 \pm 0.047 \mathrm{~mm}$ and $0.111 \pm 0.046 \mathrm{~mm}$ for the cranial/caudal, medial/lateral and anterior/posterior directions, respectively. The maximum error $(0.180 \mathrm{~mm})$ was found in the anterior/posterior direction, the Z-axis of the RSA. The $95 \%$ confidence interval of this maximum error was used as a pessimistic estimate of the accuracy of RSA for migration of the glenoid using one endoprosthetic marker and was estimated to be $0.35 \mathrm{~mm}$. This was used as the threshold level for all three directions.
Loosening was defined as a progressive migration of the glenoid component which exceeded the level of accuracy of RSA during follow-up. ${ }^{20}$

\section{Results}

Radiological assessment. Of the 48 patients, 40 (83\%) demonstrated radiolucency, of some grade, in one or more of the areas. Radiologically, 13 glenoid components $(27 \%)$ were loose. Of these, five $(10 \%)$ were considered to be loose because of a progressive radiolucency, while the remaining eight $(17 \%)$ demonstrated a shift of the component. Figure 1 shows the distribution of different grades of radiolucency in the various areas. We found an uneven distribution of radiolucency in the five areas. In $22 \%$ a small radiolucent line was seen postoperatively at the 
cement-bone interface of the superior and inferior rim of the glenoid component, whereas at follow-up more extensive and progressive radiolucencies were seen inferiorly.

RSA. The translations of the keel marker located in the glenoid component, as determined by RSA, are shown in Figure 2. Most translations occurred in the cranial/caudal and medial/lateral directions. In three patients, the total migration of the keel marker was from 1.2 to $5.5 \mathrm{~mm}$. These components were considered to be loose since their migration exceeded the RSA threshold of $0.35 \mathrm{~mm}$ in one or more directions. In one patient, a woman with osteoarthritis, the translation in the medial/lateral direction was more than the threshold level of $0.35 \mathrm{~mm}$ at the two-year follow-up. Since the migration of the component stabilised and had not progressed further by follow-up at three years the component was considered to be stable. In another patient, a woman with seropositive rheumatoid arthritis, no migration of the glenoid component was found. The radiolucencies seen on the AP radiographs of these five patients are shown in Figure 2. In one patient, the glenoid component was considered to be radiologically loose. In the remainder, only minimal radiolucencies were observed.

\section{Discussion}

The use of radiolucency as a criterion for loosening of the glenoid component has its disadvantages. Because of the oblique orientation of the glenoid fossa, measurements of radiolucency using plain radiographs are prone to error. ${ }^{6,812}$ Standardisation of the patient's position, in order to minimise these errors, is also difficult because of scapular mobility and individual anatomical variation. Assessment of the progression of radiolucent lines is especially prone to error due to a change of position of the scapula on serial radiographs. ${ }^{12}$ Since the true relationship between radiolucency and loosening is not clear, results will depend heavily on the definition of loosening. Without a standardised scoring system, different authors have used various definitions for radiological loosening of the glenoid component. This makes a comparison of the results difficult. Furthermore, the uneven distribution of radiolucencies described in our study, adds to the difficulties. If loosening is defined as a complete radiolucency of a certain grade in all regions, its frequency may be underestimated.

In our study, RSA has proven to be applicable for measuring migration of the glenoid component. The method, however, requires some practical experience when applied to such studies. It was especially difficult to obtain RSA radiographs with a sufficient number of markers in view for measurement. A total of three early follow-up examinations had to be discarded because of poor quality. The images in three of the patients in the RSA study group showed that loosening is a process which begins soon after surgery. Although three glenoid components were shown to be loose by RSA, only the most severe case had corresponding signs of radiological loosening. This discrepancy could be due to the positioning of the patient when taking a true AP radiograph, as well as difficulty in the detection and classification of radiolucent lines. It is possible that only gross loosening, with an apparent shift of the endoprosthesis or severe radiolucencies, can be detected by plain radiography. Since these signs generally need time to develop, we question the validity of standard radiographs in the assessment of early loosening of cemented glenoid components. No specific combination of radiolucent lines, or other radiological signs, was found to predict loosening of the glenoid component more accurately than in the RSA group.

We conclude that RSA should be the technique of choice in order to identify early loosening of glenoid components. It is imperative to acquire more knowledge about such loosening and its mechanism since these are major longterm issues for total shoulder replacement. The rate of loosening of glenoid components, as opposed to erosion of the glenoid in hemiarthroplasty, adds to the debate as to whether or not the glenoid should be resurfaced. If the rate of loosening in our small RSA group is representative of the whole patient population, it would argue against the use of a glenoid component.

The presence of progressive radiolucencies at the inferior pole corresponds to the findings of Amstutz et al. ${ }^{1}$ This may hold a clue for the mechanism behind the loosening process. We suggest that the largely cranial force exerted on the humeral head applies shear and rotation to the glenoid component (Fig. 1). This not only causes a shear force at the cement-bone interface, but will also produce a tensile force on the inferior part of this interface. Stress studies have shown that cement is less capable of withstanding tensile forces, ${ }^{21}$ when compared with shear or compression, and this would facilitate loosening in the inferior region. The endpoint of this mechanism would be the 'rocking horse $^{22}$ glenoid component. Future RSA studies should include three tantalum markers in the glenoid component in order to identify rotational changes which form part of the loosening process.

Although none of the authors have received or will receive benefits for personal or professional use from a commercial party related directly or indirectly to the subject of this article, benefits have been or will be received but are directed solely to a research fund, foundation, educational institution, or other non-profit institution with which one or more of the authors is associated.

\section{References}

1. Amstutz HC, Thomas BJ, Kabo JM, Jinnah RH, Dorey FJ. The Dana total shoulder arthroplasty. J Bone Joint Surg [Am] 1988;70-A:1174-82.

2. Barrett WP, Franklin JL, Jackins SE, Wyss CR, Matsen FA III. Total shoulder arthroplasty. J Bone Joint Surg [Am] 1987;69-A:86572.

3. Boyd AD, Thomas WH, Scott RD, Sledge CB, Thornhill TS. Total shoulder arthroplasty versus hemiarthroplasty: indications for glenoid resurfacing. J Arthroplasty 1990;5:329-36.

4. Brenner BC, Ferlic DC, Clayton ML, Dennis DA. Survivorship of unconstrained total shoulder arthroplasty. J Bone Joint Surg [Am] 1989;71-A:1289-96. 
5. Torchia ME, Cofield RH, Settergren CR. Total shoulder arthroplasty with the Neer prosthesis: long-term results. J Shoulder Elbow Surg 1997;6:495-505

6. Cofield RH. Total shoulder arthroplasty with the Neer prosthesis. $J$ Bone Joint Surg [Am] 1984;66-A:899-906.

7. Kelly IG, Foster RS, Fisher WD. Neer total shoulder replacement in rheumatoid arthritis. J Bone Joint Surg [Br] 1987;69-B:723-6.

8. Neer CS II, Watson KC, Stanton FJ. Recent experience in total shoulder replacement. J Bone Joint Surg [Am] 1982;64-A:319-37.

9. Kelly IG. Unconstrained shoulder arthroplasty in rheumatoid arthritis. Clin Orthop 1994;307:94-102.

10. Aliabadi P, Weissman BN, Thornhill T, Nikpoor N, Sosman JL. Evaluation of a nonconstrained total shoulder prosthesis. AJR Am J Roentgenol 1988;151:1169-72.

11. Wallace AL, Phillips RL, MacDougal GA, Walsh WR, Sonnabend DH. Resurfacing of the glenoid in total shoulder arthroplasty: a comparison, at a mean of five years of prostheses inserted with and without cement. J Bone Joint Surg [Am] 1999;81-A:510-8.

12. Havig MT, Kumar A, Carpenter W, Seiler JG III. Assessment of radiolucent lines about the glenoid: an in vitro radiographic study. $J$ Bone Joint Surg [Am] 1997;79-A:428-32.

13. Selvik G. Roentgen stereophotogrammetry: a method for the study of the kinematics of the skeletal system. Acta Orthop Scand 1989;232Suppl:1-51.
14. Kärrholm J, Borssén B, Löwenhielm G, Snorrason F. Does early micromotions of femoral stem prostheses matter? 4-7-year stereoradiographic follow-up of 84 cemented hip prostheses. J Bone Joint Surg [Br] 1994;76-B:912-7.

15. Ryd L, Albrektsson BEJ, Carlsson L, et al. Roentgen stereophotogrammetric analysis as a predictor of mechanical loosening of knee prostheses. J Bone Joint Surg [Br] 1995;77-B:377-83.

16. Amstutz HC, Sew Hoy AL, Clarke IC. UCLA anatomic total shoulder arthroplasty. Clin Orthop 1981;155:7-20.

17. MacDonald DA. The shoulder and elbow. In: Pynsent PB, Fairbank JC, Carr A, eds. Outcome measures in orthopaedics. Oxford: Butterworth Heinemann, 1993:144-73.

18. Vrooman HA, Valstar ER, Brand GJ, et al. Fast and accurate automated measurements in digitized stereophotogrammetric radiographs. J Biomech 1998;31:491-8.

19. Valstar ER. Digital roentgen stereophotogrammetry. Thesis, 2001.

20. Mjöberg B, Brismar J, Hansson LI, et al. Definition of endoprosthetic loosening: comparison of arthrography, scintigraphy and roentgen stereophotogrammetry in prosthetic hips. Acta Orthop Scand 1985;56:469-73.

21. Mann KA, Werner FW, Ayers DC. Mechanical strength of the cement-bone interface is greater in shear than in tension. J Biomech 1999;32:1251-4.

22. Wirth MA, Rockwood-CA J. Complications of total shoulder replacement arthroplasty. J Bone Joint Surg [Am] 1996;78-A:60316. 